\title{
Assessing Safety and Clinical Effectiveness of New Approaches to Planning and Integrated Implementation of Full-Mouth Reconstruction
}

\author{
Fatima Dzalaeva ${ }^{1}$ Sergey Chikunov,2 ${ }^{1,2}$ Anatoly Utyuzh ${ }^{1} \quad$ Maria Mikhailova ${ }^{1}$ Marzhanat Budunova ${ }^{1}$ \\ ${ }^{1}$ Department of Prosthodontics, First Moscow State Medical \\ University, Moscow, Russian Federation \\ 2Department of Prosthodontics, Peoples Friendship University \\ (RUDN), Moscow, Russian Federation \\ Address for correspondence Fatima Dzalaeva, PhD, Department \\ of Prosthodontics, First Moscow State Medical University, Bol'shaya \\ Pirogovskaya Street, 19c1, Moscow, 119146, Russian Federation \\ (e-mail: dzalaevafati@rambler.ru).
}

Eur J Dent:2021;5:109-116

\section{Abstract}

Keywords

- aesthetic defects

- clinical effectiveness

- complications

- patient satisfaction

- prosthodontic treatment
Objectives Planning, adequate preparation, and further prosthodontic management are the most important steps in the treatment of this population of patients with dental and mandibular anomalies. The purpose of the article is the improvement of safety and clinical effectiveness of prosthodontic rehabilitation of patients after full-mouth reconstruction.

Materials and Methods A single-center open prospective nonrandomized study, examination, and treatment of 198 patients with partial or total absence of teeth were performed. Higher safety and clinical effectiveness of the treatment were found in the patients of the main group who showed a significant reduction in the rate of complications relative to the comparison group. In the main group, the number of aesthetic defects and cases of repeated prosthetics decreased in comparison with the corresponding occurrence rate of such cases in patient groups.

Results It showed statistically significantly reduction in the rates of occurrence of the complications when using the proposed algorithm: the rate of inflammation of the marginal periodontium and peri-implant tissues in the main group was 2.9 times less frequent and loosening of the supporting implants was 3.9 times less frequent. The rate of polymer chipping or abrasion in the area of the incisal edge or masticatory surface was two times lower in the main group of patients, short crowns were found 1.9 times less often, sores in the area of the dental pontic in the main group were 1.8 times less frequent than in the comparison group, and no fractures of bridgework were found in the main group. The frequency of neuralgic facial pain in the patients of the main group was 5.2 times less than in the comparison group, complaints about pain in the temporomandibular joint area were 8.4 times less frequent, increased abrasion of antagonist teeth was 3.9 times less frequent, and phonetics disorders were 8.4 times less frequent.

The effectiveness of the algorithm developed by us is also confirmed by the fact that the number of aesthetic defects and cases of the need for repeated prosthetic treatment was reduced in comparison with the corresponding frequency of such cases in groups of patients where standard approaches to prosthetics were applied.
DOI https://doi.org/ 10.1055/s-0040-1715989 ISSN 1305-7456.
(C) 2020. European Journal of Dentistry.

This is an open access article published by Thieme under the terms of the Creative Commons Attribution-NonDerivative-NonCommercial-License, permitting copying and reproduction so long as the original work is given appropriate credit. Contents may not be used for commercial purposes, or adapted, remixed, transformed or built upon. (https://creativecommons.org/licenses/by-nc-nd/4.0/)

Thieme Medical and Scientific Publishers Pvt. Ltd., A-12, 2nd Floor, Sector 2, Noida-201301 UP, India 
In particular, aesthetic defects found in the main group of patients were 2.5 times less frequent than in the comparison group, and repeated prosthetics was performed 2.7 times less frequently.

Conclusion Application of the developed comprehensive approach to planning of prosthodontic rehabilitation provides for significant reduction in the frequency of complications and aesthetic defects, as well as cases of repeated need for prosthetics.

\section{Introduction}

It is generally accepted that planning, adequate preparation, and further prosthodontic management are the most important steps in the treatment of this population of patients with dental and mandibular anomalies (DMA). Achieving the best possible outcome of treatment depend on the effectiveness of activities performed at these stages, as well as by the cooperation of specialists in various fields in the process. Despite the fact that in most cases, prosthodontic treatment of patients with edentulism is successful-in several cases complications develop ${ }^{1-3}$-which may occur at different stages of edentulism and DMA treatment. ${ }^{4-6}$

To predict the safety of an prosthodontic treatment, different authors make attempts to assess the condition of dentures taking into account the peculiarities of their design, bacterial content, the impact of occupational hazards faced by the patients on the dentures' condition, as well as the impact of prostheses on the denture-supporting tissues. ${ }^{7-9}$ The local factors of negative impact of dentures on the periodontium include their mechanical impact: first of all, the impact of pressure under the base of the denture on the oral mucosa, including on the gingival margin of the remaining teeth. In addition, injuries of the gingival margin by the clasp's retentive arm when swallowing and chewing, as well as injuries of the interdental papilla and gingival margin-which are caused by the base of the removable denture-are often developed..$^{8-12}$

In cases of suboptimal positioning of the denture support elements when using rigid lock clasps, end defects of the dental alignment may develop, while the negative impact of removable dentures is increased in the absence of occlusal contacts in the area of remaining natural teeth. ${ }^{13-15}$

The most frequent cause of damage to the oral mucosa is chronic mechanical injury, including by partial removable denture. Depending on the source of irritation and the level of the body's reactivity, the following signs can be found in the oral mucosa: catarrhal inflammation, erosions or ulcers, gingival margin and interdental papilla hypertrophy, “denture granuloma” (lobular fibroma), papillomatosis, and hyperkeratosis.

According to Van Velzen et al, the frequency of successful implantations was $91.6 \%$, but further 10-year follow-up showed that $7 \%$ of patients develop peri-implantitis. Other authors-presenting the results of prosthetic treatment over a 10-year period-indicate that depending on the type of denture and the period after its fitting, the rate of successful prosthetic treatment was from 65 to $93 \% .{ }^{11}$
Complications of the prosthodontic treatment are divided into mechanical, biological, aesthetic, functional, and others. The rate of instability of prostheses is reported at the level of 3 to $10 \%$, and the absence of their primary fixation is observed in the early period (up to 1 month after denture installation). ${ }^{1,4}$ Complications that develop during the implantation as well as during the use of dentures are divided into complications of the surgical and prosthodontic stages. In this case, surgical complications are classified as early, developing within 1 month after the implantation, as well as late, occurring more than 1 month prior to the second stage of implantation (3-6 months). ${ }^{16,17}$

In dental practice, factors contributing to denture failure include unbalanced occlusal force, smoking, bone condition, improper handling, and mistakes in denture fitting, including injury, inadequate denture design, nonobservance of oral hygiene rules, presence of concomitant diseases, in particular, diabetes mellitus, and others. ${ }^{16,18-20}$

Bacterial infection is considered to be the most important factor contributing to denture failure, and the microbiota includes Prevotella intermedia, Porphyromonas gingivalis, Aggregatibacter actinomycetemcomitans, Bacterioides forsythus, Treponema denticola, Prevotella nigrescens, Peptostreptococcus micros, Fusobacterium nucleatum, and several other microorganisms causing peri-implantitis. ${ }^{12,14,21,22}$ Bacteria are a key etiological factor in periodontal disease, so the composition of the microbiota in the areas surrounding the denture may have a significant impact on the condition of this area in the following. ${ }^{23}$ Submucosal microflora in areas with clinically healthy margins surrounding the denture is usually represented by gram-positive cocci and rod bacteria. ${ }^{24-26}$

It has been shown that inaccuracy of clinical and radiological diagnostics and errors of prosthetic treatment can lead to various complications..$^{24,25}$ Indeed, the great variety of prosthetic complications requires their comprehensive analysis to develop preventive measures and increase treatment effectiveness.

\section{Purpose of the Study}

Improvement of safety and clinical effectiveness of the prosthodontic rehabilitation of patients after full-mouth reconstruction.

\section{Materials and Methods}

\section{Study Design}

In 2017 to 2019, a single-center open prospective nonrandomized study was conducted at the premises of department 
of prosthodontics dentistry Federal State Autonomous Educational Institution of Higher Education I.M. Sechenov First Moscow State Medical University of the Ministry of Health of the Russian Federation (Sechenov University), clinic The Medicina" Roytberg's Clinic JSC, and dental clinic Art Oral Sergey Chikunov, involving examination and treatment of 216 patients in need of a full-mouth reconstruction. Within a year after the prosthodontic treatment, the occurrence of complications, aesthetic defects, repeated need for dental prosthetics, as well as subjective satisfaction of patients with the given treatment and rehabilitation measures were assessed.

\section{Study Subjects}

The study enrolled 198 patients, the mean age was $43.1 \pm 14.32$ years, including 116 males and 82 females. The criteria for inclusion in the study were as following:

- Patient age from 20 to 75 years

- Partial or total toothlessness

- Presence of the signs of increased teeth abrasion

- Dental misalignment after prior incorrect prosthodontic treatment

- Presence of the necessity of dental restoration due to functional and aesthetic indications

Exclusion criteria:

- Presence of severe somatic pathology or exacerbations of chronic diseases

- Lack of an informed consent for inclusion in the study signed by the patient

- Patients were randomized into two groups:

- Group 1 (comparison) included 92 patients who received dentures after a standard set of prosthodontic treatment.

- Group 2 (main) included 106 patients who received dentures after using the proposed interdisciplinary approach to prosthodontic rehabilitation.

\section{Methods of Patient Treatment and Rehabilitation}

Prosthodontic treatment was performed in both groups of patients. In the comparison group, prosthodontic treatment was performed using the traditional approach based on average parameters of the patient's anatomy with the use of permanent metal-ceramic dentures without taking into account the individual features of the patient, such as central-dental ratio, therapeutic position, individual hinge and orbital axis, occlusal plane, inclination of the central incisors, and bite height.

We have developed a rehabilitation system based on an interdisciplinary approach, used in the course of treatment and rehabilitation of patients of the main group. In the course of implementing this system, we take into account results of physical examination of patients to plan DMA correction. These data were obtained using a set of diagnostic methods to assess the condition not only the oral cavity but also other various systems of the body (respiratory, central nervous, cardiovascular, and musculoskeletal).
As part of the proposed set of measures to ensure dental range stability, it is proposed to use the methods of selective grinding of dental hard tissues, temporary and permanent splinting, and ceramic restorations in a new therapeutic position. The use of acrylic crowns or bridgework is recommended during temporary restorations.

In the course of implementation of the proposed approach, anatomical and functional characteristics of the dental system were studied in the course of the treatment planning, with special attention paid to the assessment of the temporomandibular joint (TMJ) function.

Examination of the patients' anamnesis involved revealing and analysis of major errors in prior prosthetic dentistry in full-mouth reconstruction patients and specifying the causes of complications of prosthodontic treatment. Specialists in related fields (otorhinolaryngologist, neurologist, psychologist, speech therapist, osteopathic physician, and cosmetologist) are consulted.

The results of the analysis make it possible to take into account various aspects, including clinical, instrumental, functional, and aesthetic. At the same time, the use of methods of condylography and cephalometry make it possible to determine the central mandibular ratio when the models are mounted into the articulator device. To achieve this, Gamma Dental software functionality is used to develop bite simulation.

The interdisciplinary approach provides for taking into account and timely correcting functional and aesthetic disorders associated with an incorrect bite in patients undergoing full-mouth restorations.

The above approach has been used in the main group of patients, with the modeling and fabrication of the dentures performed with the help of individual gamma articulators, the advantages of which include model mounting along an adjustable articulated axis, occlusal plane measurement, and gamma rotation evaluation. At the same time, wax modeling with subsequent disconnection makes it possible to obtain high functional and aesthetic results of prosthodontic rehabilitation of patients in need of a full-mouth reconstruction (-Fig. 1). The most efficient distribution of loads on the dental system is achieved, the risk of chipping of the veneering material is reduced, and oral hygiene is improved.

\section{Study Methods}

In the course of the safety assessment of the treatment given, the occurrence of complications developed within a year after the beginning of the set of the above described prosthodontic treatment and rehabilitation activities was assessed. Detection of complications and assessment of the state of the dental and mandibular system were performed using clinical examination, orthopantomography, and condylography methods. In addition, occurrence rates of aesthetic defects of the treatment and the need for repeated prosthetics was compared between the groups of patients.

The analysis of clinical effectiveness of treatment was performed according to the results of patients' survey, summing up qualitative and quantitative indices, the final evaluation was performed according to the total characteristics 
of treatment result, being "excellent," "good," "satisfactory," and "unsatisfactory" in a year after the prosthetics treatment.

\section{Statistical Analysis}

The analysis of the study results was performed using Statsoft STATISTICA 10 and Microsoft Excel 2016 software packages. The selection of the main attributes and statistical criteria for their comparison was made after studying

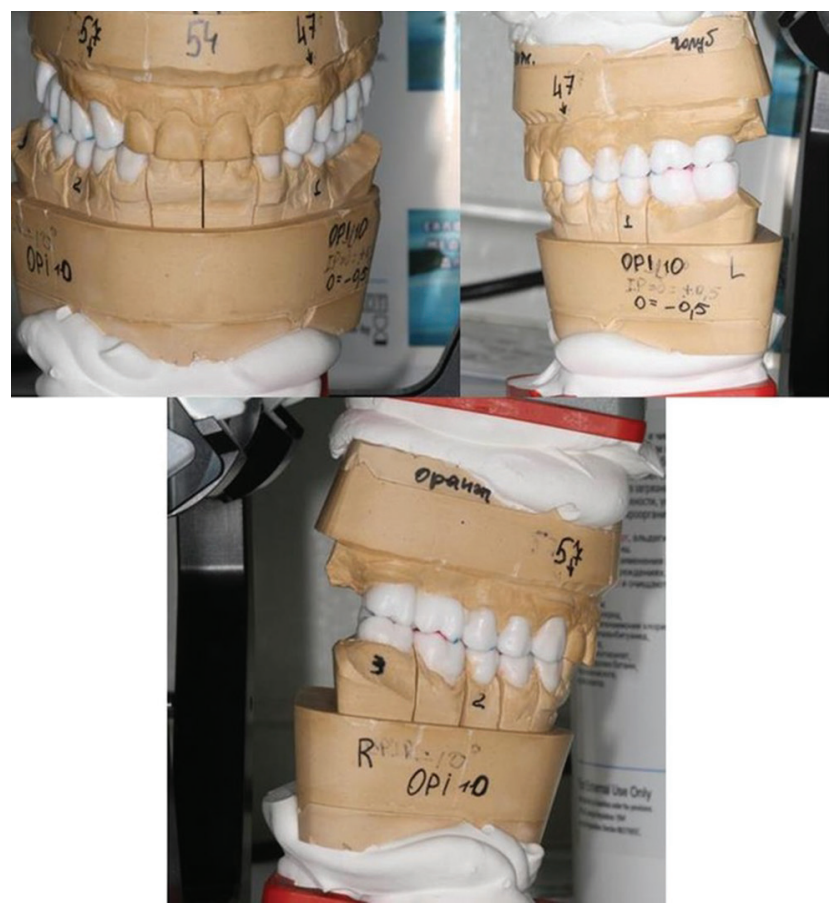

Fig. 1 An example of modeling for the fabrication of restorations performed using customized articulator devices. the distribution of the attribute and its comparison with a Gaussian distribution by using Kolmogorov-Smirnov's test.

As part of the study, qualitative parameters were compared, which were presented in the form of occurrence rates as percentages of total number of patients in the corresponding groups. Chi-square test was used for this type of intergroup differences analysis. The differences were considered statistically significant when the $p$-value was under the threshold value of statistical significance level of the null hypothesis $(\alpha)$ of 0.05 .

\section{Results}

Comparative assessment of the frequency of complications developed in patients included in the study after prosthodontic treatment showed that group 1 (comparison) had higher occurrence rate of complications than group 2 (main) for the majority of complications. As it can be seen from - Table 1, inflammation of the marginal periodontal and peri-implant tissues in group 1 was noted statistically significantly less frequent $(p<0.001)$. Loosening of supporting implants was observed in group 1 statistically significantly less frequent than in group $2(p=0.003)$.

Other complications such as polymer chipping or abrasion in the area of the incisal edge or masticatory surface, short crowns, sores in the vicinity of the dental pontic, neuralgic facial pains, pains in the temporomandibular joint area, increased abrasion of antagonist teeth, and phonetic impairment were observed statistically significantly lower in the group 2 than in group 1.

Aesthetic defects were found in the comparison group in 13 patients (14.1\%), while in the main group the value of this indicator was more than three times lower $(p=0.044)$, being 5.7\% (6 cases; - Table 2 ).

The occurrence of the need for repeated prosthetic treatment during the year was $7.6 \%$ (7 cases) in the comparison group, while in the main group there were only three

Table 1 Comparative assessment of the occurrence rate of complications after denture fitting

\begin{tabular}{|c|c|c|c|c|}
\hline \multirow[t]{2}{*}{ Complication } & \multicolumn{2}{|c|}{$\begin{array}{c}\text { Group } 1 \text { (comparison) } \\
(n=92)\end{array}$} & \multicolumn{2}{|c|}{$\begin{array}{l}\text { Group } 2 \text { (main) } \\
\quad(n=106)\end{array}$} \\
\hline & Abs. & $\%$ & Abs. & $\%$ \\
\hline $\begin{array}{l}\text { Inflammation of marginal periodontal tissue, } \\
\text { peri-implantitis }\end{array}$ & 30 & 32.6 & 12 & $11.3^{\mathrm{a}}$ \\
\hline Loosening of supporting implants & 17 & 18.5 & 5 & $4.7^{\mathrm{a}}$ \\
\hline $\begin{array}{l}\text { Polymer chipping or abrasion in the area of the incisal } \\
\text { edge or masticatory surface }\end{array}$ & 12 & 13.0 & 7 & 6.6 \\
\hline Short crowns & 18 & 19.6 & 11 & 10.4 \\
\hline Sores in the area of the dental pontic & 14 & 15.2 & 9 & 8.5 \\
\hline Bridgework fracture & 3 & 3.3 & & \\
\hline Neuralgic facial pains & 9 & 9.8 & 2 & $1.9^{\mathrm{a}}$ \\
\hline Pains in the area of the temporomandibular joint & 7 & 7.6 & 1 & $0.9^{\mathrm{a}}$ \\
\hline Increased abrasion of antagonist teeth & 10 & 10.9 & 3 & $2.8^{\mathrm{a}}$ \\
\hline Phonetic impairment & 7 & 7.6 & 1 & $0.9^{\mathrm{a}}$ \\
\hline
\end{tabular}

Abbreviation: Abs, absolute.

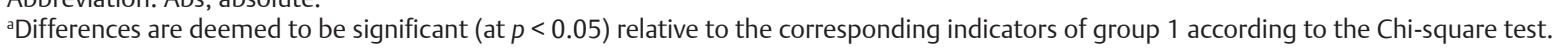


such cases (2.8\%), and the found differences were above the threshold of statistical significance ( $p=0.152$ ).

An analysis of patients' subjective assessment of the results of the prosthodontic treatment showed that the relative number of "unsatisfactory" grades in the comparison group was $20.7 \%$ (19 cases), in the main group there were only two such cases $(1.9 \%)$, the value of this indicator was statistically significant lower $(p<0.001 ;$ - Table 3$)$.
The proportion of satisfactory results also differed significantly ( $p=0.002$ ), with $46.7 \%$ (43 patients) and $18.9 \%$ (20 cases) in the comparison group and main group, respectively.

A total of 22 patients $(23,9 \%$ ) of group 1 assessed the results of treatment as good, while in the main group the value of this indicator was statistically significantly higher $(p<0.001)$, being 48 patients (45.3\%).

In the comparison group, there were eight patients (8.7\%) who assessed the treatment result as excellent, while in the

Table 2 Presence of aesthetic defects and occurrence of the need for repeated prosthetic treatment within a year after denture fitting

\begin{tabular}{|l|l|l|l|l|}
\hline \multirow{2}{*}{ Indicators } & \multicolumn{2}{|c|}{$\begin{array}{c}\text { Group 1 (comparison) } \\
(\boldsymbol{n}=92)\end{array}$} & \multicolumn{2}{c|}{$\begin{array}{c}\text { Group 2 (main) } \\
(\boldsymbol{n}=106)\end{array}$} \\
\cline { 2 - 5 } & Abs. & $\%$ & Abs. & $5.7^{\text {a }}$ \\
\hline Presence of aesthetic defects & 13 & 14.1 & 6 & 2.8 \\
\hline Need for repeated prosthetic treatment & 7 & 7.6 & 3 & \\
\hline
\end{tabular}

Abbreviation: Abs, absolute.

aDifferences are deemed to be significant (at $p<0.05$ ) relative to the corresponding indicators of group 1 according to the Chi-square test.

Table 3 Subjective assessment by patients 1 year after denture fitting

\begin{tabular}{|l|l|l|l|l|}
\hline \multirow{2}{*}{ Grade } & \multicolumn{2}{|c|}{$\begin{array}{c}\text { Group 1 (comparison) } \\
(n=92)\end{array}$} & \multicolumn{2}{c|}{$\begin{array}{c}\text { Group 2 (main) } \\
(n=106)\end{array}$} \\
\cline { 2 - 6 } & Abs. & $\%$ & Abs. & $\%$ \\
\hline Excellent & 8 & 8.7 & 36 & $48.0^{\text {a }}$ \\
\hline Good & 22 & 23.9 & 20 & $45.3^{\text {a }}$ \\
\hline Satisfactory & 43 & 46.7 & 2 & $18.9^{\text {a }}$ \\
\hline Unsatisfactory & 19 & 20.7 & $1.9^{\text {a }}$ \\
\hline
\end{tabular}

Abbreviation: Abs, absolute.

aDifferences are deemed to be significant (at $p<0.05$ ) relative to the corresponding indicators of group 1 according to the Chi-square test.

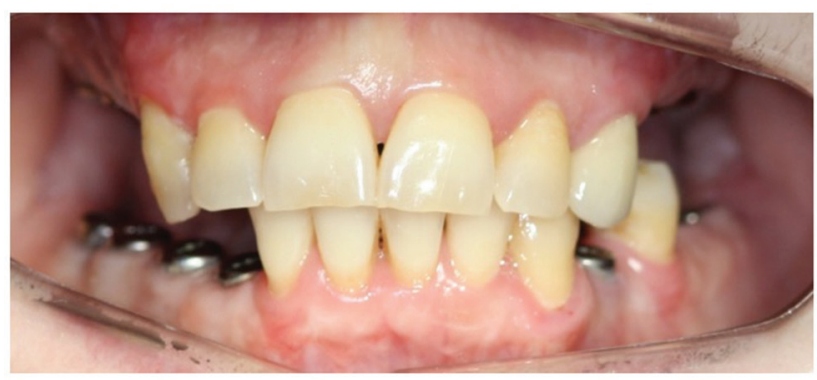

$\mathbf{A}$
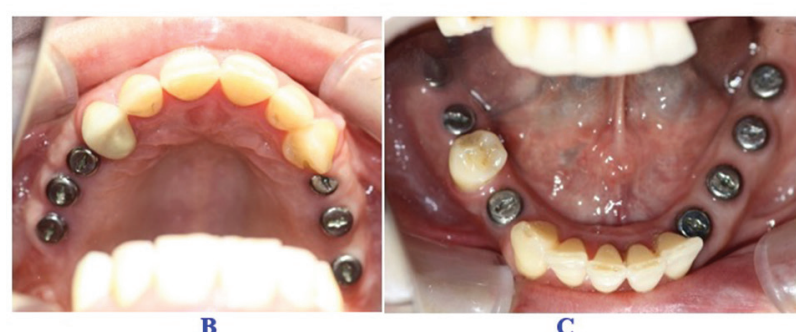

C

Fig. 2 Patient B. Intraoral photographs of teeth before treatment: (A) frontal plane; (B) occlusal surfaces of the teeth of the upper jaw; and $(\mathbf{C})$ occlusal surfaces of the teeth of the lower jaw.

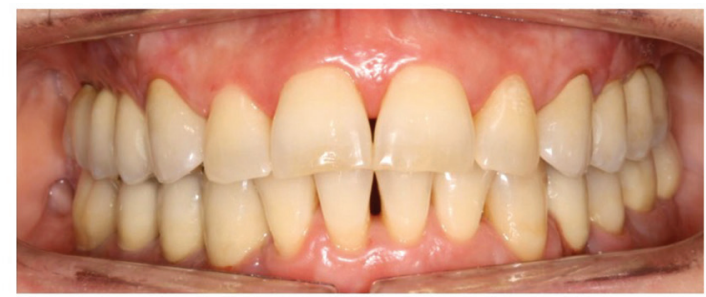

A

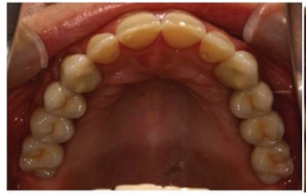

B

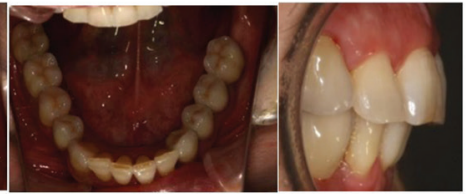

$\mathrm{C}$
Fig. 3 Patient B. The final result of treatment: (A) frontal plane; (B) the occlusal plane of the teeth of the upper jaw; (C) is the occlusal surface of the teeth of the lower jaw; (D) the depth and width of the cutting floor. 
main group the fraction of such patients was statistically significantly higher ( $p<0.001$ ), being 34.0\% (36 cases).

The result of denture treatment presented on - Figs. 2 and 3. The view before treatment is presented in -Fig. 2 .

-Fig. 3 shows the final treatment result.

\section{Discussion}

Currently, most authors consider not only the correction of deformities and malocclusion of teeth, but also the achievement of facial harmony as the main goals of prosthodontic treatment. 7,13,16,25 At the same time, experts note the increasing insistence of patients upon the state of the dental system, and the results of functional and aesthetic prosthodontic rehabilitation. ${ }^{26-28}$ Nevertheless, clinical effectiveness of the treatment of patients with DMA and edentulism in need of full-mouth reconstruction often becomes impaired due to the development of complications.

Several conservative methods for correcting joint disorders have been proposed. In clinical practice, selective grinding, acupuncture, therapeutic exercises, autogenic training, and physiotherapeutic procedures are actively used. ${ }^{9,19,20}$ Together with other specialists, patients with TMJ dysfunction are prescribed drug therapy. Most often, such patients are prescribed sedatives, antidepressants, muscle relaxants, and antidepressants. Using this approach in patients with TMJ dysfunction determines the need to use a set of functional and radiation diagnostics methods, which allow obtaining a quantitative and qualitative analysis of the systems involved in the pathological process, and developing a targeted systemic treatment, rehabilitation program with subsequent quantitative and qualitative analysis of the effectiveness of this program. ${ }^{4,17}$

To a great extent, the development of these complications is due the fact that practitioners often do not take into account facial features, the skeletal nature of mandibular deformities, functional relations between adjacent anatomical structures while being focused on bite normalization only, which negatively affects the final result of treatment. Several researchers give attention to the issue of rates of recurrence of dental and mandibular deformities, study of the causes, and mechanisms of their occurrence. ${ }^{4,6}$ However, these researches are mostly based on the limited number of patients and are limited to statement of the most frequently occurring complications without carrying out any profound analysis of the causes of their development and definition of ways and methods of prevention and treatment of such complications in a set of prosthodontic treatment and rehabilitation activities.

The data obtained in this study have shown higher safety and clinical effectiveness of treatment of these patients in the course of full-mouth reconstruction, which-in our opinionis due to careful planning of activities performed in the main group. In particular, we observed a significant reduction in the rates of occurrence of the following complications when using the proposed algorithm: the rate of inflammation of the marginal periodontium and peri-implant tissues in the main group was 2.9 times less frequent, loosening of the supporting implants was 3.9 times less frequent (statistically significantly). The rate of polymer chipping or abrasion in the area of the incisal edge or masticatory surface was two times lower in the main group of patients, short crowns were found 1.9 times less often, sores in the area of the dental pontic in the main group were 1.8 times less frequent than in the comparison group, and no fractures of bridgework were found in the main group.

The frequency of neuralgic facial pain in the patients of the main group was 5.2 times less than in the comparison group, complaints about pain in the TMJ area were 8.4 times less frequent, increased abrasion of antagonist teeth was 3.9 times less frequent, phonetics disorders were 8.4 times less frequent.

The effectiveness of the algorithm developed by us is also confirmed by the fact that the number of aesthetic defects and cases of the need for repeated prosthetic treatment was reduced in comparison with the corresponding frequency of such cases in groups of patients, where standard approaches to prosthetics were applied. In particular, aesthetic defects found in the main group of patients were 2.5 times less frequent than in the comparison group, and repeated prosthetics was performed 2.7 times less frequently.

The above-mentioned results contributed to higher clinical effectiveness of the prosthetic treatment given as well as better subjective assessment by patients who were fitted with different prosthetic designs and confirmed their satisfaction with the performed dental prosthodontic treatment on the basis of the approach suggested by us. In particular, at subjective assessment of the results of the prosthodontic treatment by patients from the main group, the number of unsatisfactory grades was 10.9 times less than in the comparison group. The percentage of satisfactory grades was 2.5 times lower, good grades 1.9 times higher, and excellent grades 3.9 times higher in comparison with corresponding grades in the group of patients who had been receiving treatment based on standard approaches to prostodonthic rehabilitation.

The data obtained by us are largely consistent with the results of other authors. For example, Fedotova ${ }^{25}$ published a retrospective assessment of the results of prosthetic treatment with full removable dentures. The author described the main complications that developed when using full removable laminar dentures: plaque on the denture (65\%), mechanical damages of the denture (38\%), sore ulcers of the mucosa at the denture base (70\%), dietary restrictions, that is, exclusion of certain products from the patients' diet (53\%), imprints of the denture edges on the mucous membrane of the denture base (43\%), phonetic impairment, in particular the difficulty of pronouncing sibilant and fricative consonants (37\%), difficulties in chewing food (28\%), and food getting under the denture (21\%). The author also pays attention to the presence of gaps around the artificial teeth (79\%), relatively low quality of the modeling (61\%) and the aesthetics of denture (46\%). ${ }^{25}$

In general, the results of the conducted studies show that the proposed multidisciplinary approach to prosthodontic rehabilitation of patients makes it possible to obtain a longterm prognosis of the condition and functioning of dentures at the stage of treatment planning. Its use in clinical practice 
is pathogenetically justified, based on taking into account the anatomical and physiological characteristics of the dental and mandibular system.

According to the performed assessment of the effectiveness of the proposed approach, the application of the developed system providing for taking into account these factors before prosthetic treatment, makes it possible to significantly reduce the frequency of complications and aesthetic defects, as well as cases of need for repeated prosthetic treatment. In our opinion, application of the developed algorithm as part of expert assessment at the stage of planning treatment of dental alignment defects contributes to significant reduction of errors in a prosthodontist's work and increase patients' satisfaction with the treatment given.

Nevertheless, it should be noted that the data presented in the literature on the complications of surgical treatment of congenital mandibular anomalies have not been systematized as of today, there is no clear algorithm of prevention of errors leading to their development, no approaches to minimization of the risk of complications at various stages of patient management have been developed yet, and there is no classification of possible complications of surgical treatment of this category of patients.

\section{Conclusion}

The obtained results testify that application of the methodology of the interdisciplinary approach becomes the key factor of high safety and clinical effectiveness of prosthodontic treatment in full-mouth reconstruction patients. It has been established that application of the developed algorithm of diagnostics and planning of prosthodontic rehabilitation contributes to statistically significantly lower (relative to the comparison group) levels of complication frequency, better aesthetic results of treatment, statistically significantly higher satisfaction of patients with the treatment and rehabilitation given in this population of patients within a year after the beginning of treatment.

The key stage in the implementation of our proposed approach is a complete and consistent examination of fullmouth reconstruction patients. In the course of solving the problems that emerge in the course of planning the set of necessary therapeutic and rehabilitative activities, it is necessary to supplement the traditional approach with diagnostic protocols and treatment of several other disorders, in particular, aesthetic defects. At planning of prosthetic and prosthodontic treatment, it is necessary to establish cause-and-effect relationship of the specified disorders with the incorrect bite.

The obtained data confirm the necessity to take into account the etiopathogenesis and individual anatomical and physiological characteristics of full-mouth reconstruction patients with DMA, carry out a comprehensive diagnosis with the involvement of specialists in multiple fields in the process of treatment while planning treatment and rehabilitation activities.

\section{Data Availability}

Data will be available on request.

\section{Funding}

None.

\section{Conflict of Interest}

None declared.

\section{References}

1 Wolford LM. Comprehensive post orthognathic surgery orthodontics: complications, misconceptions, and management. Oral Maxillofac Surg Clin North Am 2020;32(1):135-151

2 Romanos GE, Javed F. Platform switching minimises crestal bone loss around dental implants: truth or myth? J Oral Rehabil 2014;41(9):700-708

3 Ali DA. Patient satisfaction in dental healthcare centers. Eur J Dent 2016;10(3):309-314

4 Schminke B, Vom Orde F, Gruber R, Schliephake H, Bürgers R, Miosge $N$. The pathology of bone tissue during peri-implantitis. J Dent Res 2015;94(2):354-361

5 Ketoff S, Sigaux N, Raberin M, Bouletreau P. [Dental complications during orthodontic preparation and orthognathic surgery]. Orthod Fr 2018;89(2):137-144

6 Van Gorp G, Bormans N, Vanham I, Willems G, Declerck D. Orthodontic treatment recommendation and expected adverse reactions in patients with a history of dental trauma: a survey among general dentists, paediatric dentists, and orthodontic specialists. Int J Paediatr Dent 2020;30(3):360-369

7 Wang CW, Yu SH, Mandelaris GA, Wang HL. Is periodontal phenotype modification therapy beneficial for patients receiving orthodontic treatment? An American Academy of Periodontology best evidence review. J Periodontol 2020;91(3):299-310

8 Rossi-Fedele G, Franciscatto GJ, Marshall G, Gomes MS, Dogramac EJ. Endodontic complications associated with orthodontic temporary anchorage devices: A systematic review of human studies. Aust Endod J 2020;46(1):115-122

9 Saakian ShKh, Kalamkarov AE. The analysis of changes in bone at orthopedic treatment of patients with defects of tooth alignments with use the dental implants. Rus J Dentist 2014;2:13-16

10 Fonseca EPD. Multivariate method to identify inequalities in oral healthcare access. Eur J Dent 2018;12(4):475-479

11 van Velzen FJ, Ofec R, Schulten EA, Ten Bruggenkate CM. 10 -year survival rate and the incidence of peri-implant disease of 374 titanium dental implants with a SLA surface: A prospective cohort study in 177 fully and partially edentulous patients. Clin Oral Implants Res 2015;26(10):1121-1128

12 Park JH, Park JJ, Papademetriou M, Suri S. Anterior open bite due to idiopathic condylar resorption during orthodontic retention of a Class II Division 1 malocclusion. Am J Orthod Dentofacial Orthop 2019;156(4):555-565

13 Gallone M, Robiony M, Bordonali D. Bruno G, De Stefani A, Gracco A. Multidisciplinary treatment with a customized lingual appliance for an adult patient with severe Class III malocclusion and multiple missing teeth. Am J Orthod Dentofacial Orthop 2019;156(3):401-411

14 Gao JY, Yu XQ. Efficacy of orthodontic and orthognathic treatment for oral and maxillofacial deformities. Medicine (Baltimore) 2019;98(39):e17324

15 Yumashev AV, Utyuzh AS, Volchkova IR, Mikhailova MV, Kristal EA. The influence of mesodiencephalic modulation on the course of postoperative period and osseointegration quality in case of intraosseus dental implantation. Ind J Sci Tech 2016;9:1-8

16 Chiu G, Chang C, Roberts WE. Interdisciplinary treatment for a compensated Class II partially edentulous malocclusion: 
orthodontic creation of a posterior implant site. Am J Orthod Dentofacial Orthop 2018;153(3):422-435

$17 \mathrm{Oz} \mathrm{AZ}$, Ciger S. Health of periodontal tissues and resorption status after orthodontic treatment of impacted maxillary canines. Niger J Clin Pract 2018;21(3):301-305

18 Ahn JC, Lee JH, Yoon JH, Lee JY, Kim JH. Interdisciplinary treatment of a patient with multiple missing teeth and periodontitis. Am J Orthod Dentofacial Orthop 2018;153(2):278-289

19 Lam R, Goonewardene MS, Allan BP, Sugawara J. Success rates of a skeletal anchorage system in orthodontics: a retrospective analysis. Angle Orthod 2018;88(1):27-34

20 Ravaghi V, Al-Hammadi Z, Landes D, Hill K, Morris AJ. Inequalities in orthodontic outcomes in England: treatment utilisation, subjective and normative need. Community Dent Health 2019;36(3):198-202

21 Dalago HR, Schuldt Filho G, Rodrigues MA, Renvert S, Bianchini MA. Risk indicators for Peri-implantitis: a cross-sectional study with 916 implants. Clin Oral Implants Res 2017;28(2):144-150

22 Mombelli A, Müller N, Cionca N. The epidemiology of peri-implantitis. Clin Oral Implants Res 2012;23(6, Suppl 6):67-76
23 Belibasakis GN, Charalampakis G, Bostanci N, Stadlinger B. Peri-implant infections of oral biofilm etiology. Adv Exp Med Biol 2015;830:69-84

24 Choi YY. Relationship between orthodontic treatment and dental caries: results from a national survey. Int Dent J 2020;70(1):38-44

25 Fedotova EA. Retrospective assessment of prosthetic care results with complete loss of teeth. Abstract of the thesis of Candidate of Medical Sciences, St. Petersburg

26 Gurdan Z, Szalma J. Evaluation of the success and complication rates of self-drilling orthodontic mini-implants. Niger J Clin Pract 2018;21(5):546-552

27 Rakhshan H, Rakhshan V. Pain and discomfort perceived during the initial stage of active fixed orthodontic treatment. Saudi Dent J 2015;27(2):81-87

28 Nishi SE, Basri R, Alam MK. Uses of electromyography in dentistry: an overview with meta-analysis. Eur J Dent 2016;10(3):419-425 